\title{
Necessary Optimality Condition for Vector Optimization Problems in Infinite-dimensional Linear Spaces Xuanwei Zhou
}

\author{
Dept.of Basic Courses.Zhejiang Shuren University.Hangzhou 310015, China \\ wdzxw@163.com
}

\begin{abstract}
Keywords: infinite-dimensional linear space, theorem of alternative, necessary optimality condition, vector optimization problem.
\end{abstract}

\begin{abstract}
In this paper, the concept of preconvexlike functions is introduced. Then, under the convexity, the vector optimization problem in Hausdorff topological vector spaces is studied. A theorem of the alternative is established. By use of the theorem of the alternative, a necessary optimality condition for vector optimization problems in infinite-dimensional linear spaces are obtained.
\end{abstract}

\section{Introduction}

Optimality conditions are a key topic in optimization: they are useful not only for identifying solutions of the problem, but they are also crucial in designing numerical methods for approximating the solutions. It is well known that theorems of alternative are very useful for optimality conditions in optimization problems (see [1]-[16]). Giannessi ([6]-[7]) stressed the concept of image of constrained extremum problems, which has turned out to be a powerful tool for investigating optimality conditions in optimization.

On the other hand, convexity and generalized convexity are playing some central roles in optimization. Fan [5] introduced the concept of convexlike condition that was of interest for extensions of the von Neumann minimax theorem. Later, Jeyakumar ([10]-[12]) presented the concept of subconvexlike function, which generalized Fan's convexlikeness. Recently, theorems of the alternative were generally employed to derive many result in optimization for convexlike or subconvexlike functions ([1], [3], [6]-[9],[10]-[14]). Among these, Gwinner and Oettli ([9]) studied functions defined in arbitrary nonempty sets; Jeyakumar ([10]-[11]) and Giannessi (Ref. [6]) considered the situations of Banach spaces, normed spaces, or finite-dimensional Euclidean spaces; Jeyakumar ([12]) and $\mathrm{Li} \mathrm{([13])} \mathrm{attained} \mathrm{some} \mathrm{theorems} \mathrm{of} \mathrm{the} \mathrm{alternative} \mathrm{for} \mathrm{subconvexlike}$ functions and optimality conditions for vector optimization problems in locally convex Hausdorff vector topological spaces.

In this paper, first we introduce the concept of preconvexlike functions. Then, a theorem of the alternative for preconvexlike functions in Hausdorff topological vector spaces is established. By use of the theorem of the alternative, we obtain a necessary optimality condition for vector optimization problems in Hausdorff topological vector spaces.

\section{Definitions and Lemmas}

Let $X$ be a real topological vector space, let $Y *$ be the topology dual of $X$. A subset $K$ of $X$ is said to be a convex cone if

$$
\alpha x_{1}+\beta x_{2} \in K, \forall x_{1}, x_{2} \in K, \forall \alpha, \beta \geq 0 .
$$

A positive cone is a convex cone with apex at the origin.

A real topological vector space $X$ with a convex cone is said to be an ordered topological vector space. The partial order on $X$ is defined by $x_{1} \leq_{K} x_{2}$, iff $x_{2}-x_{1} \in K, x_{1} \leq_{\text {int } K} x_{2}$, iff $x_{2}-x_{1} \in$ int $K$, where int $K$ is the topological interior of $K$.

Setting $K^{*}=\left\{\xi \in X^{*}:\langle\xi, x\rangle \geq 0, \forall x \in K\right\}, K^{*}$ is said to be the dual cone of the positive cone K. 
Lemma 2.1. ([4]) Let $X$ be a Housdorff topological vector space. If $\xi \in K^{*} \backslash\{O\}$ and $x_{0} \in \operatorname{int} K$, then $\left\langle\xi, x_{0}\right\rangle>0$.

Let $X$ be a real topological vector space, let $Y$ be an ordered topological vector space with a positive cone $K_{1}$. A function $f: X \rightarrow Y$ is said to be $K_{1}$-convex on $X$ iff, $\forall x_{1}, x_{2} \in X, \forall \alpha \in(0,1), \alpha f\left(x_{1}\right)+(1-\alpha) f\left(x_{2}\right)-f\left(\alpha x_{1}+(1-\alpha) x_{2}\right) \in K_{1}$.

Definition 2.1. ([5]) Let $X$ be a real topological vector space, let $Y$ be an ordered topological vector space with a positive cone $K_{1}$. A function $f: X \rightarrow Y$ is said to be $K_{1}$-convexlike on $X$ iff, $\forall x_{1}, x_{2} \in X, \forall \alpha \in(0,1), \exists x_{3} \in X$ such that

$$
\alpha f\left(x_{1}\right)+(1-\alpha) f\left(x_{2}\right)-f\left(x_{3}\right) \in K_{1} .
$$

Definition 2.2. ([10]) Let $X$ be a real topological vector space, let $Y$ be an ordered topological vector space with a positive cone $K_{1}$, and $E$ a nonempty subset of $Y$. A function $f: X \rightarrow Y$ is said to be $\left(K_{1}, E\right)$-subconvexlike on $X$ iff $\exists$ a bounded $E$-valued function $u: X \rightarrow Y$, for $\forall x_{1}, x_{2} \in X, \forall \alpha \in(0,1), \exists x_{3} \in X$ such that

$$
\varepsilon u+\alpha f\left(x_{1}\right)+(1-\alpha) f\left(x_{2}\right)-f\left(x_{3}\right) \in K_{1} .
$$

In particular, if $E=Y$, then $f$ is said to be $K_{1}$-subconvexlike on $X$.

In this paper, we introduce the definition of preconvexlikeness as follows.

Definition 2.3. Let $X$ be a real topological vector space, let $Y$ be an ordered topological vector space with a positive cone $K_{1}$. A function $f: X \rightarrow Y$ is said to be $K_{1}$-preconvexlike on $X$ iff, $\forall x_{1}, x_{2} \in X, \forall \alpha \in(0,1), \exists x_{3} \in X$ and $\exists \tau>0$, such that

$$
\alpha f\left(x_{1}\right)+(1-\alpha) f\left(x_{2}\right)-\tau f\left(x_{3}\right) \in K_{1} .
$$

We will establish a theorem of the alternative in the following.

Let $X$ be a real topological vector space, let $Y, Z, W$ be Hausdorff topological vector spaces with respectively positive cones $K_{1}, K_{2}, K_{3}$, which are supposed to have nonempty interiors (say) int $K_{1}$, int $K_{2}$, int $K_{3}$

Definition 2.4. ([14]). Let $F: X \rightarrow Y, M \subset Y$. For each neighborhood $R$ of the origin $O$ of $Y$, if there exists a neighborhood $U$ of the origin of $X$ such that, $\forall x \in\left(x^{\prime}+U\right) \cap D(F), F(x) \in\left(F\left(x^{\prime}\right)+R\right)+M$, then $F$ is said to be $M$-continuous at $x^{\prime}$, where $D(F)$ denotes the domain of $F$.

Theorem 2.1. Assume that the functions $F: X \rightarrow Y, G: X \rightarrow Z$ and $H: X \rightarrow W$ satisfy the conditions (a) $f=(F, G, H)$ is $\left(K_{1} \times K_{2} \times\{O\}, Y \times Z \times\{O\}\right)$-preconvex -likeon $X$; (b) the Slater condition(SC1):

$\left(x^{\prime}, y^{\prime}, z^{\prime}, w^{\prime}\right) \in G r(f):=\{(x, F(x), G(x), H(x)): x \in X\}$ such that $z^{\prime}<_{\text {int } K_{1}} 0$ and $w^{\prime}=0$;

(c) the continuity condition: $F$ is $\left(-K_{1}\right)$-continuous at $x^{\prime}$ and $G$ is $\left(-K_{2}\right)$ -

continuous at $x^{\prime}$; (d) the inner point condition: for all neighborhoods $\mathrm{R}$ of the origin of $Y$, for all neighborhoods $S$ of the origin of $Z$, there exists a neighborhood $T$ of the origin of $W$ such that $T \subset H(U)$, where

$$
U=\left\{x \in X: F(x) \in F\left(x^{\prime}\right)+R-K_{1}, G(x) \in G\left(x^{\prime}\right)+S-\operatorname{int} K_{2}\right\} .
$$

Then, one and only one of the systems (S1) and (S2) has solutions:

(S1) $\exists x_{0} \in X$ such that $F\left(x_{0}\right)<_{\text {int } K_{1}} O, G\left(x_{0}\right) \leq_{K_{2}} O$, and $H\left(x_{0}\right)=O$.

(S2) $\exists(\xi, \eta, \zeta) \in\left\{K_{1} * \backslash\{O\} \times K_{2} * \times W^{*}\right\}$

such that

$$
\langle\xi, F(x)\rangle+\langle\eta, G(x)\rangle+\langle\zeta, H(x)\rangle \geq 0, \quad \forall x \in X .
$$

Proof. If $x$ and $(\xi, \eta, \zeta)$ are the solutions of the systems of (S1) and (S2) respectively, then by Lemma 2.1,

$$
0 \leq\langle\xi, F(x)\rangle+\langle\eta, G(x)\rangle+\langle\zeta, H(x)\rangle \leq\langle\xi, F(x)\rangle<0 .
$$


This is a contradiction. Therefore, (S1) and (S2) exclude each other.

We suppose now that (S1) is impossible; we aim to show that (S2) holds. Let $C=\bigcup_{t>0}(t B+P)$, where $B=\{(F(x), G(x), H(x)): x \in X\}$ and $P=\left\{\right.$ int $\left.K_{1} \times K_{2} \times\{O\}\right\}, \quad O$ is the origin of $W$. At first, It can be easily proved that $C$ is convex.

Now, we are going to show that $\operatorname{int} C \neq \varnothing$. From the Slater condition (SC1), $\exists \tilde{x} \in X$ such that $G\left(x^{\prime}\right)<_{\text {int } K_{2}} O, H\left(x^{\prime}\right)=O$. Therefore, $\exists$ a neighborhood $S$ of the origin $O$ of $Z$ such that

$$
-G\left(x^{\prime}\right)+S \subset \operatorname{int} K_{2} \text {. }
$$

On the other hand, if we take $\bar{y} \in \operatorname{int} K_{1}$ such that $\bar{y}-F\left(x^{\prime}\right) \in \operatorname{int} K_{1}$, then $\exists$ a neighborhood $R$ of the origin $O$ of $Y$ such that $(R+R$ is also a neighborhood of the origin of $Y$ )

$$
\bar{y}-F\left(x^{\prime}\right)+R \in \operatorname{int} K_{1} \text {. }
$$

Let $T$ be the same as in condition (d). Then, we can prove that

$$
(\bar{y}+R) \times S \times T \subset C \text {. }
$$

In fact, $\forall(y, z, w) \in(\bar{y}+R) \times S \times T$, from (d), $\exists x \in U$ such that $w=H(x)$, where $U$ is the same as in condition (d). By (c), there exists $y_{1}, z_{1}$ such that

$$
y_{1}=F(x) \in F\left(x^{\prime}\right)-R-K_{1}, \quad z_{1}=G(x) \in G\left(x^{\prime}\right)-S-K_{2} .
$$

Therefore, combining (2) and (3), we have

$$
y-y_{1} \in(\bar{y}+R)-\left(F\left(x^{\prime}\right)-R-K_{1}\right) \subset \bar{y}-F\left(x^{\prime}\right)+R+R-K_{1} \subset \operatorname{int} K_{1}+K_{1}=\operatorname{int} K_{1} .
$$

Similarly, by (1) and (3), we have $z-z_{1} \in \operatorname{int} K_{2}$. Hence,

$$
(y, z, w)=\left(y_{1}, z_{1}, w\right)+\left(y-y_{1}, z-z_{1}, O\right) \subset B+\text { int } K_{1} \times \text { int } K_{2} \times\{O\} \subset C .
$$

Therefore, $(\bar{y}+R) \times S \times T \subset C$ and soint $C \neq \varnothing$.

Finally, since (S1) has no solution, we have $O \notin C$. Otherwise, if $O \in C$, then $\exists x_{0} \in X$ such that

$$
t\left(F\left(x^{0}\right), G\left(x^{0}\right), H\left(x^{0}\right)\right)+(\hat{v}, \hat{z}, O)=O,
$$

for some positive number $t>0$, where $\hat{y} \in \operatorname{int} K, \hat{z} \in K_{1}$. Then, we would have

$$
F\left(x_{0}\right)=-\frac{1}{t} \hat{y}<_{\text {int } K_{1}} O, G\left(x_{0}\right)=-\frac{1}{t} \hat{z}<_{K_{2}} O, H\left(x_{0}\right)=O,
$$

which implies that $x_{0}$ is a solution of (i), contradicting the assumption. Therefore, $C$ is convex, int $C \neq \varnothing$, and $O \notin C$.

By the separation theorem of convex sets of a topological vector space, $\exists \mu=(\xi, \eta, \zeta) \in(Y \times Z \times W)^{*} \subset Y^{*} \times Z^{*} \times W^{*}$ such that

$$
\langle\mu, t(F(x), G(x), H(x))\rangle+\langle\mu,(y, z, O)\rangle \geq 0,
$$

for $\forall x \in X, \forall(y, z, O) \in P=$ int $K_{1} \times K_{2} \times\{O\}, \forall t>0$. From (4), we see that

$$
\mu \in K_{1} * \times K_{2} * \times W^{*} \text {. }
$$

Again, from (4), for any given $(y, O, O) \in P=\operatorname{int} K_{1} \times K_{2} \times\{O\}$ and for a sequence $\left\{s_{n}\right\}$ of positive numbers with $s_{n} \rightarrow 0(n \rightarrow \infty)$, we have

$$
\langle\mu,(F(x), G(x), H(x))\rangle+\left\langle\mu,\left(s_{n} y, O, O\right)\right\rangle \geq 0, \forall x \in X .
$$

Letting $n \rightarrow \infty$, we have

$$
\langle\xi, F(x)\rangle+\langle\eta, G(x)\rangle+\langle\zeta, H(x)\rangle \geq 0, \forall x \in X .
$$

On the other hand, if $\xi=O$, then

from which we deduce that

$$
\langle\eta, G(x)\rangle+\langle\zeta, H(x)\rangle \geq 0, \forall x \in X,
$$

$$
0 \leq\langle\eta, G(\tilde{x})\rangle+\langle\zeta, H(\tilde{x})\rangle=\langle\eta, G(\tilde{x})\rangle<0,
$$

since by the Slater condition (SC1), $G(\tilde{x}) \in \operatorname{int} K, \eta \in K^{*}$. This is a contradiction. Therefore, $\xi \neq O$. So, we have proved that, if (S1) has no solution, then (S2) has a solution. 


\section{Vector Optimization Problems}

In this section, we discuss vector optimization problems. Our preconvexlikeness and theorems of the alternative will be used. We consider the program

(VP) $\min f(x)$, s.t. $-g(x) \in K_{2}, h(x)=0$,

where $f: X \rightarrow Y, g: X \rightarrow Z, h: X \rightarrow W$.

Definition 3.1. $x_{0} \in X$ is said to be a weakly efficient solution of (VPI) if $\nexists x \in D$ such that $f(x) \leq_{K_{1}} f\left(x_{0}\right)$, where $D=\left\{x \in X: g(x) \leq_{K_{2}} O, h(x)=O\right\}$.

Definition 3.2. Let $X, Y$ be Hausdorff topological vector spaces, and let $\varphi: X \rightarrow Y$. Let $\bar{X} \in X$ and $x \in X$ be a nonzero vector. The Hadamard directional derivative of $\varphi$ at $\bar{X}$ along the vector $x$, denoted by $\varphi_{\bar{x}}^{\prime}(x)$, is given by the following limit if it exists: $\varphi_{\bar{x}}^{\prime}(x)=\lim _{\substack{t \rightarrow 0^{+} \\ u \rightarrow x}}(\varphi(\bar{x}+t u)-\varphi(\bar{x})) / t$. We say that $\varphi$ is Hadamard directional differentiable if $\varphi_{\bar{x}}^{\prime}(x)$ exists for any $x \in X$.

Definition 3.3. Let $S$ be a nonempty set in $X$, and let $\bar{x} \in$ clS. The cone of tangents of $S$ at $\bar{x}$, denoted by $T$, is the set of all directions $d$ such that $d=\lim _{k \rightarrow \infty} \lambda_{k}\left(x_{k}-\bar{x}\right)$, where $\lambda_{k}>0$, $x_{k} \in S$ for each $k$, and $x_{k} \rightarrow \bar{x}$.

Theorems 3.1. Let $S$ be a nonempty set in $X$, and let $\bar{x} \in S$. Furthermore, suppose that $f: X \rightarrow Y$ is Hadamard directional differentiable at $\bar{x}$. If $\bar{X}$ is a weakly efficient solution of the problem to minimize $f(x)$ subject to $x \in S$, then $F_{0} \cap T=\varnothing$, where $F_{0}=\left\{x \in X: f^{\prime}{ }_{\bar{x}}(x)<_{\text {int } K_{1}} O\right\}$ and $T$ is the cone of tangents of $S$ at $\bar{x}$.

Proof. Suppose that $F_{0} \cap T \neq \varnothing$. Let $x^{\prime} \in F_{0} \cap T$. From $x^{\prime} \in T$, we have $x^{\prime}=\lim _{k \rightarrow \infty} \lambda_{k}\left(x_{k}-\bar{x}\right)$, where $\lambda_{k}>0, x_{k} \in S$ for each $k$, and $x_{k} \rightarrow \bar{x}$. Set $u_{k}=\lambda_{k}\left(x_{k}-\bar{x}\right)$, then $x_{k}=\bar{x}+t_{k} u_{k}$ where $t_{k}=1 / \lambda_{k} \rightarrow 0, u_{k} \rightarrow x^{\prime}, x_{k} \in S$.

Since $f: X \rightarrow Y$ is differentiable at $\bar{x}$, by definition of differentiable,

$$
f^{\prime} \bar{x}_{\bar{x}}\left(x^{\prime}\right)=\lim _{\substack{t_{k} \rightarrow 0^{+} \\ u_{k} \rightarrow x^{\prime}}}\left(f\left(\bar{x}+t_{k} u_{k}\right)-f(\bar{x})\right) / t_{k} \text {. }
$$

From $x^{\prime} \in F_{0}$, we have that $f_{\bar{x}}^{\prime}\left(x^{\prime}\right) \in-$ int $K_{1}$. There exists $k$ such that $\left(f\left(\bar{x}+t_{k} u_{k}\right)-f(\bar{x})\right) / t_{k} \in-\operatorname{int} K_{1}$. Thus, $f\left(x_{k}\right)-f(\bar{x}) \in-\operatorname{int} K_{1}$. This contradicts that $\bar{x}$ is a weakly efficient solution of (VP). Therefore, $F_{0} \cap T=\varnothing$.

Theorem 3.2. Let $X$ be Hausdorff topological vector space and let $Y, Z, W$ be Hausdorff topological vector spaces with respectively positive cones $K, K_{1}, K_{2}$, which are supposed to have nonempty interiors (say) int $K$, int $K_{1}$, int $K_{2}$, and $f: X \rightarrow Y, g: X \rightarrow Z, h: X \rightarrow W$. Let $\bar{x} \in D$ be a weakly efficient solution of (VP), where $D=\left\{x \in X: g(x) \leq_{K_{2}} O, h(x)=O\right\}$. Suppose that $f, g, h$ are Hadamard directional differentiable at $\bar{x}$. Suppose that the constraint qualification $T \supset G^{\prime} \cap H_{0}$ holds true, where $T$ is the cone of tangents of $D$ at $\bar{x}$, and $G^{\prime}=\left\{x: g_{\bar{x}}^{\prime}(x) \leq_{K_{2}}-g(\bar{x})\right\}, H_{0}=\left\{x: h_{\bar{x}}^{\prime}(x)=O\right\}$. Furthermore, assume that the functions $f^{\prime}{ }_{\bar{x}}, g_{\bar{x}}^{\prime}, h_{\bar{x}}^{\prime}$ satisfy the conditions: (a) $\varphi$ is $\left(K_{1} \times K_{2} \times\{O\}, Y \times Z \times\{O\}\right)$-preconvexlike on $X$, where $\varphi(x)=\left(f_{\bar{x}}^{\prime}(x), g_{\bar{x}}^{\prime}(x)+g(\bar{x}), h_{\bar{x}}^{\prime}(x)\right)$;

(b) the Slater condition (SC2): $\left(x^{\prime}, y^{\prime}, z^{\prime}, w^{\prime}\right) \in\left\{\left(x, f_{\bar{x}}^{\prime}(x), g_{\bar{x}}^{\prime}(x), h_{\bar{x}}^{\prime}(x)\right): x \in D\right\}$ such that $z^{\prime}<_{\text {int } K_{2}}-g(\bar{x})$ and $w^{\prime}=0$; (c) the continuity condition: $f_{\bar{x}}^{\prime}(x)$ is $\left(-K_{1}\right)$-continuous at $x^{\prime}$ and $g_{\bar{x}}^{\prime}(x)+g(\bar{x})$ is $\left(-K_{2}\right)$-continuous at $x^{\prime}$; (d) the inner point condition: for all neighborhoods $R$ of the origin of $Y$, for all neighborhoods $S$ of the origin of $Z, \exists$ a neighborhood $T$ of the origin of 
W such that $T \subset H(U)$, where

$U=\left\{x \in X: f^{\prime}{ }_{\bar{x}}(x) \in f^{\prime}{ }_{\bar{x}}\left(x^{\prime}\right)+R-K_{1}, g_{\bar{x}}^{\prime}(x) \in g_{\bar{x}}^{\prime}\left(x^{\prime}\right)+S-\operatorname{int} K_{2}\right\}$.

Then, there exists $(\xi, \eta, \zeta) \in\left\{K_{1} * \backslash\{O\} \times K_{2} * \times W^{*}\right\}$ such that

$\left\langle\xi, f_{\bar{x}}^{\prime}(x)\right\rangle+\left\langle\eta, g_{\bar{x}}^{\prime}(x)\right\rangle+\left\langle\zeta, h_{\bar{x}}^{\prime}(x)\right\rangle \geq 0(\forall x \in X)$ and $\langle\eta, g(\bar{x})\rangle=0$.

Proof. Since $\bar{x} \in D$ is a weakly efficient solution of (VP), by Theorem 3.1, we get $F_{0} \cap T=\varnothing$. By the supposition, we have $F_{0} \cap G^{\prime} \cap H_{0}=\varnothing$, that is, the system $f^{\prime}{ }_{\bar{x}}(x)<_{\text {int } K_{1}} O, g_{\bar{x}}^{\prime}(x)+g(\bar{x}) \leq_{K_{2}} O$, and $h_{\bar{x}}^{\prime}(x)=O$ has no solution. According to the assumption, we have that $f_{\bar{x}}^{\prime}(x), g_{\bar{x}}^{\prime}(x)+g(\bar{x}), h_{\bar{x}}^{\prime}(x)$ satisfy the conditions (a)-(d) in Theorem 2.1. Therefore, by Theorem 2.1, there exists $(\xi, \eta, \zeta) \in\left\{K_{1} * \backslash\{O\} \times K_{2} * \times W^{*}\right\}$ such that

$$
\left\langle\xi, f^{\prime}{ }_{\bar{x}}(x)\right\rangle+\left\langle\eta, g_{\bar{x}}^{\prime}(x)+g(\bar{x})\right\rangle+\left\langle\zeta, h_{\bar{x}}^{\prime}(x)\right\rangle \geq 0, \forall x \in X .
$$

Taking $x=O$ in the above formula, we get $\langle\eta, g(\bar{x})\rangle \geq 0$. On the other hand, from $\eta \in K_{2} *$ and $g(\bar{x}) \leq_{K_{2}} O$, we get $\langle\eta, g(\bar{x})\rangle \leq 0$. So,

$$
\langle\eta, g(\bar{x})\rangle=0 \text {. }
$$

Therefore, combining (1) and (2), we have

$\left\langle\xi, f_{\bar{x}}^{\prime}(x)\right\rangle+\left\langle\eta, g_{\bar{x}}^{\prime}(x)\right\rangle+\left\langle\zeta, h_{\bar{x}}^{\prime}(x)\right\rangle \geq 0(\forall x \in X)$ and $\langle\eta, g(\bar{x})\rangle=0$.

This completes the proof.

\section{Summary}

A preconvexlike function is obviously a presubconvexlike function. However, after we have the concept of preconvexlikeness, this sequence of convexity or generalized convexity (convex, convexlike, subconvexlike, preconvexlike, and presubconvexlike) is more interesting. Sometimes, however, preconvexlikeness is better than presubconvexlikeness in some sense. But if we change preconvexlikeness to presubconvexlikeness, then we have to change "Hausdorff topological vector spaces" to "locally convex Hausdorff topological vector spaces" and then, of course, we have to change the proofs.

On the other hand, any real-valued function is a preconvexlike function. And we have defined a nonnegative or nonpositive function that is valued in a topological vector space by means of a positive cone. Moreover, any nonnegative or nonpositive function is preconvexlike. These are some advantages of preconvexlike functions.

\section{References}

[1]M.Arana-Jiménez, G.Ruiz-Garzón, A.Rufián-Lizana and R.Osuna-Gómez: Weak efficiency in multiobjective variational problems under generalized convexity, J. Glob. Optim. Vol.52(2012), p. $109-121$.

[2]M.B.Asadi, M.Soleimani-damaneh: Infinite Alternative Theorems and Nonsmooth Constraint Qualification Conditions, Set-Valued and Variational Analysis, Vol.20, (2012), p.551-566.

[3]M.S.Bazaraa: A Theorem of the Alternative with Application to Convex Program- ming, Optimality, Duality, and Stability, Journal of Mathematical Analysis and Applications, Vol.41(1973), p. 701-715 .

[4]K.Deiming: Nonlinear Functional Analysis,Springer Verlag,Berlin, Germany,1985. [5]K.Fan: Minimax Theorems, Proceedings of the National Academy of Sciences of the USA, Vol.39(1953), p. 42-47.

[6] F.Giannessi: Theorems of the Alternative for Multifunctions with Application toOptimization: General Results, Journal of Optimization Theory and Applications, Vol. 55(1987), p. 233-256 .

[7] F.Giannessi: Theorems of the Alternative and Optimality Conditions, Journal of Optimization Theory and Applications, Vol.42(1984), p. 331-365 .

[8] B.D.Graven, J.Gwinner and V.Jeyakumar: Nonconvex Theorems of the Alternative and 
Minimization, Optimization, Vol. 18(1987), p. 151-163 .

[9] J.Gwinner, W. Oettli: Theorems of the Alternative and Duality for Inf-Sup Problems, Mathematics of Operations Research, Vol.19(1994), p. 238-256 .

[10]V. Jeyakumar: Convexlike Alternative Theorems and Mathematical Programming, Optimization, Vol.16(1985), p. 643-652 .

[11]V. Jeyakumar: A General Farkas Lemma and Characterization of Optimality for a Nonsmooth Program Involving Convex Processes, Journal of Optimization Theory and Applications, Vol.55(1987), p. 449-467 .

[12] V. Jeyakumar and J.Gwinner: Inequality Systems and Optimization, Journal of Mathematical Analysis and Applications, Vol.159(1991), p. 51-71 .

[13]Z. Li: The Optimality Conditions of Differentiable Vector Optimization Problems, Journal of Mathematical Analysis and Applications, Vol.201(1996), p. 35-43 .

[14]D. T. Luc: On Duality Theorems in Multiobjective Programming, Journal of Optimization Theory and Applications, Vol.48(1984), p. 557-582 .

[15]V.Michel:Theorems of the Alternative for Multivalued Mappings and Applications to Mixed Convex Concave Systems of Inequalities, Set-Valued Analysis, Vol.18(2010), p. 601-616 .

[16]E.Ning, W.Song and Y.Zhang: Second order sufficient optimality conditions in vector optimization, Journal of Global Optimization, Vol.54(2012), p.537-549. 